\title{
GROUPS WHOSE NONLINEAR IRREDUCIBLE CHARACTERS SEPARATE ELEMENT ORDERS OR CONJUGACY CLASS SIZES
}

\author{
MARIAGRAZIA BIANCHI, DAVID CHILLAG, AND EMANUELE PACIFICI
}

\begin{abstract}
A class function $\varphi$ on a finite group $G$ is said to be an order separator if, for every $x$ and $y$ in $G \backslash\{1\}, \varphi(x)=\varphi(y)$ is equivalent to $x$ and $y$ being of the same order. Similarly, $\varphi$ is said to be a class-size separator if, for every $x$ and $y$ in $G \backslash\{1\}, \varphi(x)=\varphi(y)$ is equivalent to $\left|C_{G}(x)\right|=\left|C_{G}(y)\right|$. In this paper, finite groups whose nonlinear irreducible complex characters are all order separators (respectively, class-size separators) are classified. In fact, a more general setting is studied, from which these classifications follow. This analysis has some connections with the study of finite groups such that every two elements lying in distinct conjugacy classes have distinct orders, or, respectively, in which disctinct conjugacy classes have distinct sizes.
\end{abstract}

\section{INTRODUCTION}

In a paper appeared in $1985([6])$, P. Fitzpatrick studied the class of finite groups which satisfy the following property: every two elements of the group lying in distinct conjugacy classes have distinct orders. The conclusion of that work, also obtained by W. Feit and G. M. Seitz in [5], is that $S_{3}$ (the symmetric group on three objects) is the unique nonabelian group belonging to this class.

In the same spirit, a well known conjecture states that $S_{3}$ is the unique nontrivial finite group in which distinct conjugacy classes have distinct sizes. A major step in this context is the work of J. P. Zhang in [15], and of R. Knörr et al. in [14], showing that the above statement is true in the class of solvable groups.

Now, we say that a class function $\varphi$ of a finite group is an order function if it takes the same value on nonidentity elements having the same order, whereas $\varphi$ is a class-size function if it takes the same value on nonidentity elements lying in conjugacy classes of the same size. With this terminology, the results in the two paragraphs above can be stated as follows. Let $G$ be a nonabelian finite group, and assume that every irreducible character of $G$ is an order function, or that $G$ is solvable, and every irreducible character of $G$ is a class-size function. Then $G$ is isomorphic to $S_{3}$ (here we also take into account [8, Corollary 3]).

In the present paper, we consider a situation in which from one point of view these assumptions are sharpened, and from another one they are relaxed. We say that an order function $\varphi$ of $G$ is an order separator if, for every $x$ and $y$ in $G \backslash\{1\}, \varphi(x)=\varphi(y)$ implies that $x$ and $y$ have the same order. Similarly, a

2000 Mathematics Subject Classification. 20C15.

The second author would like to thank the Department of Mathematics at the Univesity of Milano for the support and hospitality. 
class-size function $\varphi$ is a class-size separator if, for every $x$ and $y$ in $G \backslash\{1\}$, $\varphi(x)=\varphi(y)$ implies that $x$ and $y$ lie in conjugacy classes of the same size. Given that, we can state the following results, a proof of which is given in Section 2.

Theorem A. Let $G$ be a nonabelian finite group, and assume that every nonlinear irreducible character of $G$ is an order separator. Then one of the following holds.

(a) $G$ is isomorphic to $Q_{8}$ (the quaternion group of order 8 ).

(b) $G$ is isomorphic to $S_{3}$.

(c) $G$ is isomorphic to $\operatorname{AGL}\left(1,2^{\alpha}\right)$ (the affine group of dimension 1 over the field with $2^{\alpha}$ elements), where $2^{\alpha}-1$ is a prime.

In particular, $G$ has a unique nonlinear irreducible character. Conversely, if $G$ is as in (a), (b) or (c), then the unique nonlinear irreducible character of $G$ is an order separator.

Theorem B. Let $G$ be a nonabelian finite group, and assume that every nonlinear irreducible character of $G$ is a class-size separator. Then one of the following holds.

(a) $G$ is an extraspecial 2-group.

(b) $G$ is isomorphic to $\operatorname{AGL}\left(1, p^{\alpha}\right)$ (the affine group of dimension 1 over the field with $p^{\alpha}$ elements).

In particular, $G$ has a unique nonlinear irreducible character. Conversely, if $G$ is as in (a) or (b), then the unique nonlinear irreducible character of $G$ is a class-size separator.

In both cases $S_{3}$ is in the list, but other groups also occur. Moreover, we observe that the groups appearing in the conclusions of Theorem A appear in those of Theorem B as well.

Theorems A and B follow as special cases of the main result in this paper. Given a finite group $G$, consider an equivalence relation $\mathcal{R}$ on the set $G \backslash\{1\}$ satisfying the following property: if two elements in $G \backslash\{1\}$ generate the same subgroup of $G$, then they lie in the same $\mathcal{R}$-equivalence class (we say that such an equivalence relation is rational; see the explanation after Definition 1.3). Also, we define the concepts of $\mathcal{R}$-function and $\mathcal{R}$-separator in the obvious way (see 1.1 and 1.2 ). In this setting, the following holds.

Main Theorem. Let $G$ be a nonabelian finite group, $\mathcal{R}$ a rational equivalence relation on $G \backslash\{1\}$, and assume that every nonlinear irreducible character of $G$ is an $\mathcal{R}$-separator. Then $G^{\prime}$ is a minimal normal subgroup of $G$, and one of the following holds.

(a) $G$ is an extraspecial 2-group.

(b) $G$ is a Frobenius group with kernel $G^{\prime}$ (which is an elementary abelian p-group for a suitable prime $p$ ) and cyclic complement.

Also, some properties of finite groups whose nonlinear irreducible characters are all $\mathcal{R}$-functions are derived in Lemma 1.4 .

It may be worth observing that, in the setting of the Main Theorem and assuming $Z(G) \neq 1$, the relation $\mathcal{R}$ is necessarily the one which identifies two nonidentity 
elements of $G$ if and only if they lie in conjugacy classes of the same size (call $\mathcal{S}$ this relation). However, this does not hold in general when $Z(G)=1$. In fact, consider a Frobenius group with elementary abelian kernel of order 9 and cyclic complement of order 4 . We can define an equivalence relation $\mathcal{R}$ on the nonidentity elements of this group so that the assumptions of the Main Theorem are satisfied, but which is definitely not $\mathcal{S}$.

To close with, the proof of the Main Theorem depends on the classification of finite simple groups. More precisely, the proof of Theorem 1.6, in which the solvability of groups satisfying the assumptions of the Main Theorem is established, uses the classification via an application of [5, Theorem B]. Also, throughout the whole discussion, every abstract group is tacitly assumed to be finite. The notation is standard; if $G$ is a group, we shall use the symbol $\operatorname{Lin}(G)$ to denote the set of linear characters of $G$.

\section{1. $\mathcal{R}$-FUNCTIONS AND $\mathcal{R}$-SEPARATORS}

We start by giving the precise definition of some concepts mentioned in the Introduction.

Definition 1.1. Let $G$ be a group, $\mathcal{R}$ an equivalence relation on the set $G \backslash\{1\}$, and $\varphi$ a class function of $G$. We say that $\varphi$ is an $\mathcal{R}$-function if, for every $x, y$ in $G \backslash\{1\}, x \mathcal{R} y$ implies $\varphi(x)=\varphi(y)$.

Definition 1.2. In the same setting as above, we say that a class function $\varphi$ is an $\mathcal{R}$-separator if, for every $x, y$ in $G \backslash\{1\}, x \mathcal{R} y$ is equivalent to $\varphi(x)=\varphi(y)$.

It is clear that an $\mathcal{R}$-separator is also an $\mathcal{R}$-function. Moreover, the existence of an $\mathcal{R}$-separator for a group $G$ implies that each $\mathcal{R}$-equivalence class is a union of conjugacy classes of $G$.

Definition 1.3. Let $G$ be a group, and let $\mathcal{G}$ be the equivalence relation on $G \backslash\{1\}$ defined as follows: for $x, y$ in $G \backslash\{1\}, x \mathcal{G} y$ holds if and only if $x$ and $y$ generate the same subgroup of $G$. We say that an equivalence relation $\mathcal{R}$ on $G \backslash\{1\}$ is rational if it contains $\mathcal{G}$.

Recall that, if $G$ is a group, a character $\chi$ of $G$ is said to be rational if all the values taken by $\chi$ are rational numbers. It is well known that $\chi$ is rational if and only if, for every $x$ and $y$ in $G$ such that $\langle x\rangle=\langle y\rangle$, we have $\chi(x)=\chi(y)$ (a partial proof can be found in $[\mathbf{1 2}, 5.22])$. This should motivate the choice of the word rational in Definition 1.3. It is also worth recalling that an element $x$ of $G$ is said to be rational if every character of $G$ takes a rational value on $x$, and it is well known that $x$ is rational if and only if it is conjugate to every $y$ in $G$ such that $\langle x\rangle=\langle y\rangle$ (see [13,22.15] and the comment following it). Finally, the group $G$ is said to be rational if every character of $G$ is rational (which is of course equivalent to the fact that every element of $G$ is rational).

The following lemma derives a number of properties of groups whose nonlinear irreducible characters are all $\mathcal{R}$-functions. 
Lemma 1.4. Let $G$ be a nonabelian group, $\mathcal{R}$ a rational equivalence relation on $G \backslash\{1\}$, and assume that every nonlinear irreducible character of $G$ is an $\mathcal{R}$-function. Then the following conclusions hold.

(a) Every element of $G^{\prime}$ is rational in $G$.

(b) If $x, y \in G \backslash\{1\}$ are $\mathcal{R}$-equivalent and not conjugate, then, for every $\chi$ in $\operatorname{Irr}(G) \backslash \operatorname{Lin}(G)$, we get $\chi(x)=\chi(y)=0$. In particular, if $x$ is in $G^{\prime} \backslash\{1\}$, then every element of $G \backslash\{1\}$ which is $\mathcal{R}$-equivalent to $x$ is conjugate to $x$.

(c) Every nonlinear irreducible character of $G$ is rational.

(d) Let $\chi$ be in $\operatorname{Irr}(G) \backslash \operatorname{Lin}(G)$, let $\mathcal{C}$ be an $\mathcal{R}$-equivalence class, and let $\chi_{\mathcal{C}}$ denote the value which $\chi$ takes on $\mathcal{C}$. If $M$ is a subgroup of $G$ whose nonidentity elements are in $\mathcal{C}$, then $\chi(1)-\chi_{\mathcal{C}}$ is divisible by $|M|$.

(e) Let $N$ be a nontrivial normal subgroup of $G$ whose nonidentity elements lie in an $\mathcal{R}$-equivalence class $\mathcal{C}$. Then $N \backslash\{1\}$ is a conjugacy class of $G$, so that $N$ is minimal normal in $G$, and it is an elementary abelian p-group for a suitable prime $p$. Moreover, we get $N \backslash\{1\}=\mathcal{C}$. Finally, if $\chi$ is a nonlinear irreducible character of $G$ whose kernel does not contain $N$, then $\chi(1)=-\chi_{\mathcal{C}} \cdot|\mathcal{C}|$, where $\chi_{\mathcal{C}}$ denotes the value which $\chi$ takes on $\mathcal{C}$.

(f) Let $G$ be nonsolvable. Then $G$ is a rational group, and either $G$ is perfect, or $G / G^{\prime}$ is an elementary abelian 2 -group.

Proof. Let $x$ be in $G \backslash\{1\}$, and let $y \in G$ be such that $\langle x\rangle=\langle y\rangle$. We have $x \mathcal{G} y$, whence $x \mathcal{R} y$. Now, if $\chi$ is $\operatorname{in} \operatorname{Irr}(G) \backslash \operatorname{Lin}(G)$, we get $\chi(x)=\chi(y)$. If $x$ is in $G^{\prime} \backslash\{1\}$, then the same holds also for every linear character of $G$, thus (a) is proved.

Assume that $x, y \in G \backslash\{1\}$ are $\mathcal{R}$-equivalent and not conjugate. Then there exists $\lambda$ in $\operatorname{Lin}(G)$ such that $\lambda(x) \neq \lambda(y)$ (otherwise all irreducible characters of $G$ would take the same value on $x$ and $y)$. If $\chi$ is in $\operatorname{Irr}(G) \backslash \operatorname{Lin}(G)$, then $\lambda \chi$ must take the same value on $x$ and $y$, and so $\lambda(x) \chi(x)=\lambda(y) \chi(y)$ holds. Now, the equality $\chi(x)=\chi(y)$ forces $\chi(x)$ to be 0 . Since, by the Second Orthogonality Relation $([\mathbf{1 2}, 2.18])$, it can not happen that every nonlinear irreducible character of a (nonabelian) group $H$ vanishes on an element of $H^{\prime}$, the second claim of (b) follows as well.

Next, for every $\chi$ in $\operatorname{Irr}(G) \backslash \operatorname{Lin}(G)$ and $x$ in $G$, we aim to show that $\chi(x)$ is a rational number. If it is not, then there exists $y$ in $G$ such that $\langle x\rangle=\langle y\rangle$ and $x$ is not conjugate to $y$. Nevertheless, $x$ and $y$ are $\mathcal{R}$-equivalent, so (b) yields $\chi(x)=0$. We reached a contradiction, and (c) is proved.

We now prove claim (d). By [12, Problem 3.8], there exist integers $a$ and $b$ such that $\chi \downarrow_{M}=a 1_{M}+b \rho_{M}$, where $\rho_{M}$ denotes the regular character of $M$. If $b=0$, then $M \leq \operatorname{ker} \chi$ and the claim follows, so we may assume $b \neq 0$. Let $x$ be in $M \backslash\{1\}$. We get $\chi_{\mathcal{C}}=a+b \rho_{M}(x)=a$, whence $\chi \downarrow_{M}=\chi_{\mathcal{C}} \cdot 1_{M}+b \rho_{M}$. Now, $\chi(1)=\chi_{\mathcal{C}}+b \rho_{M}(1)=\chi_{\mathcal{C}}+b|M|$, and the claim follows in this case as well.

As for (e), we first observe that there exists $\xi \operatorname{in} \operatorname{Irr}(G) \backslash \operatorname{Lin}(G)$ such that $N$ does not lie in ker $\xi$. In fact, assume this is false. Then, taking into account that the intersection of all the kernels of irreducible characters of a group is trivial, we 
can find $\lambda$ in $\operatorname{Lin}(G)$ such that $N \not \leq \operatorname{ker} \lambda$, so there exists $x$ in $N$ such that $\lambda(x) \neq 1$. Now, if $\psi$ is in $\operatorname{Irr}(G) \backslash \operatorname{Lin}(G)$, we get $\lambda \psi(x)=\lambda \psi(1)$, and this yields a contradiction. As in (d), we have $\xi \downarrow_{N}=\xi_{\mathcal{C}} \cdot 1_{N}+b \rho_{N}$, where $b$ is not 0 . Therefore, every nonprincipal irreducible character of $N$ is a constituent of $\xi \downarrow_{N}$, and Clifford's Theorem $([\mathbf{9}, 19.3(\mathrm{c})])$ implies that $G$ generates a single orbit in its natural action on $\operatorname{Irr}(N) \backslash\left\{1_{N}\right\}$. Assume now that $\operatorname{Irr}(N) \backslash \operatorname{Lin}(N)$ is not empty. As the elements of $\operatorname{Irr}(N) \backslash \operatorname{Lin}(N)$ lie in a single $G$-orbit, they are forced to have the same degree, thus $N$ is solvable (see $[\mathbf{1 2}, 12.6]$ ). But this implies that $\operatorname{Lin}(N) \backslash\left\{1_{N}\right\}$ is not empty, so that all the irreducible characters of $N$ must be linear. This contradicts our assumptions, whence $N$ does not have any nonlinear irreducible character, and so it is abelian. An application of the Brauer Permutation Lemma ([9, 18.5(c)]) yields now that $N \backslash\{1\}$ is a conjugacy class, whence $N$ is minimal normal in $G$, and it is an elementary abelian $p$-group for a suitable prime $p$. Also, if $\mathcal{C}$ is not contained in $N$, then (by (b)) every nonlinear irreducible character of $G$ vanishes on $N \backslash\{1\}$, so that $G / N$ must be abelian. But now every nonlinear irreducible character of $G$ vanishes on $G^{\prime} \backslash\{1\}$, and this is a contradiction, as mentioned in the proof of (b). Hence $\mathcal{C}=N \backslash\{1\}$. Finally, let $\chi$ be a nonlinear irreducible character of $G$ such that $N \not \leq$ ker $\chi$. By Clifford's Theorem we get $\chi \downarrow_{N}=z\left(\lambda_{1}+\lambda_{2}+\cdots+\lambda_{t}\right)$, where $z$ is a positive integer and $\lambda_{1}, \lambda_{2}, \ldots, \lambda_{t}$ are the elements of $\operatorname{Irr}(N) \backslash\left\{1_{N}\right\}$ (so $t=|N|-1=|\mathcal{C}|$ ). Now we get $\chi_{\mathcal{C}}=-z$, and also the last claim of (e) follows.

We move now to (f). Suppose that $G$ is not a rational group. Then, in view of (c), there must be a linear character of $G$ which is not rational, hence not real. So $G$ is a nonreal group, and every nonreal irreducible character of $G$ is linear. We are in a position to apply Theorem 1.4 in $[3]$ and conclude that $G$ is solvable. Let now $G$ be nonsolvable (hence rational). If $G$ is not perfect, then $G / G^{\prime}$ is a nontrivial abelian rational group, whence it is an elementary abelian 2 -group. This completes the proof of (f), and of the lemma.

The remaining part of this section will focus on groups whose nonlinear irreducible characters are all $\mathcal{R}$-separators. Lemma 1.5, which follows at once by the previous result, will be a key tool in the proof of Theorem 1.6, where the solvability of such groups is established. After that, we shall prove the Main Theorem stated in the Introduction.

Lemma 1.5. Let $G$ be a nonabelian group, $\mathcal{R}$ a rational equivalence relation on $G \backslash\{1\}$, and assume that every nonlinear irreducible character of $G$ is an $\mathcal{R}$ separator. Also, let $N$ be a nontrivial normal subgroup of $G$ such that $G / N$ is nonabelian. Then all the conclusions in (e) of Lemma 1.4 hold. Moreover, if $N$ is properly contained in $G^{\prime}$, then $G^{\prime} / N$ is a minimal normal subgroup of $G / N$.

Proof. As $G / N$ is nonabelian, there exists a nonlinear irreducible character $\chi$ of $G$ whose kernel contains $N$, so that $\chi$ is constant on $N$. This implies that $N \backslash\{1\}$ lies in an $\mathcal{R}$-equivalence class, therefore the assumptions of Lemma 1.4(e) are fulfilled. Moreover, let $N$ be properly contained in $G^{\prime}$, and let $M$ be a normal subgroup of $G$ such that $N \leq M<G^{\prime}$. Applying to $M$ the first claim of this lemma, we get that $M$ is minimal normal in $G$, and this forces $M$ to coincide with $N$. 
Occasionally, we shall use Lemma 1.4 and Lemma 1.5 without any reference.

Theorem 1.6. Let $G$ be a (nonabelian) group, $\mathcal{R}$ a rational equivalence relation on $G \backslash\{1\}$, and assume that every nonlinear irreducible character of $G$ is an $\mathcal{R}$ separator. Then $G$ is solvable.

Proof. For a proof by contradiction, we shall assume that $G$ is nonsolvable, and therefore a rational group (Lemma 1.4(f)). By [5, Theorem B], every nonabelian composition factor of $G$ is isomorphic to an alternating group $A_{n}$ (with $n \geq 5$ ), or to one of the following groups: $\operatorname{PSp}(4,3), \operatorname{Sp}(6,2), \mathrm{SO}^{+}(8,2), \operatorname{PSL}(3,4), \operatorname{PSU}(4,3)$.

Let $N$ be a normal subgroup of $G$ which is properly contained in $G^{\prime}$ (we allow $N=1$ only if $G^{\prime}$ is minimal normal in $G$ ). If $N \neq 1$, then $N$ is an elementary abelian $p$-group for some prime $p$, and $G^{\prime} / N$ is a (nonsolvable) chief factor of $G$. Otherwise, $G^{\prime}$ is itself a nonsolvable chief factor of $G$. In any case we have $G^{\prime} / N=S_{1} \times S_{2} \times \cdots \times S_{m}$, where the $S_{i}$ are isomorphic nonabelian simple groups. Denoting $S_{1}$ by $S$, we have that $S$ is isomorphic to one of the groups in the list of the above paragraph.

Our next step will be to show that $C_{G / N}\left(G^{\prime} / N\right)$ is trivial. Let $A$ be the (normal) subgroup of $G$ which contains $N$ and such that $A / N=C_{G / N}\left(G^{\prime} / N\right)$. We have that $A / N$ intersects $(G / N)^{\prime}$ trivially, thus $A / N$ is a central subgroup of $G / N$ (in fact, $A / N=Z(G / N))$. Hence $G / A$, which is isomorphic to $(G / N) /(A / N)$, is nonabelian, so either $A$ is trivial (which can obviously happen only if $N$ is trivial), or $A$ is an abelian minimal normal subgroup of $G$. We have to deal with the latter situation. If in this case $N$ is nontrivial, then $A$ is forced to coincide with $N$ and we are also done. Finally, assume $N=1$ and $A \neq 1$, and choose $t$ in $G^{\prime} \backslash\{1\}$ and $z$ in $A \backslash\{1\}$. If $\psi \in \operatorname{Irr}(G) \backslash \operatorname{Lin}(G)$ is such that $A \leq \operatorname{ker} \psi$, we have $\psi(z t)=\psi(t)$, whence $z t$ and $t$ are $\mathcal{R}$-equivalent. But $z t$ and $t$ can not be conjugate in $G$, and this violates (b) of Lemma 1.4.

In the sequel we shall use the fact that, according to [7, Corollary 1] and [4], $S$ has an irreducible character of $q$-defect zero for a suitable prime $q$. Namely, if $n \geq 5$, then $A_{n}$ has an irreducible character of $q$-defect zero for every prime $q$ greater than 3 dividing its order, the groups $\operatorname{PSp}(4,3)$ and $\operatorname{PSU}(4,3)$ both have irreducible characters of defect zero for all the prime divisors of their orders, and the groups $\operatorname{Sp}(6,2), \mathrm{SO}^{+}(8,2), \operatorname{PSL}(3,4)$ all have irreducible characters of 5 -defect zero.

As the next step, let us prove that $G^{\prime} / N$ is simple (that is, $m=1$ ). Suppose $m \geq 2$, and let $\chi_{i} \in \operatorname{Irr}\left(S_{i}\right)$ be of $q$-defect zero. Define $\varphi$ to be the irreducible character of $G^{\prime} / N$ given by $\chi_{1} \times \chi_{2} \times \cdots \times \chi_{m}$. Then all the $G / N$-conjugates of $\varphi$ have $q$-defect zero in $G^{\prime} / N$, and so they vanish on every $q$-singular element of $G^{\prime} / N$ (see [12, 8.17]). If $\vartheta$ is an irreducible character of $G / N$ whose restriction to $G^{\prime} / N$ has $\varphi$ as a constituent, we have that $\vartheta \downarrow_{G^{\prime} / N}$ is a multiple of a sum of $G / N$-conjugates of $\varphi$, and therefore $\vartheta$ vanishes on every $q$-singular element of $G^{\prime} / N$. Let $N u \in S_{1}$ be of order $q$, and $N y \in S_{2}$ of prime order $r$ different from $q$. Then $N v:=N u y$ has order $q r$, and so $\vartheta(N u)=\vartheta(N v)=0$. Now, regarding $\vartheta$ as a character of $G$ by inflation, we get $\vartheta(u)=\vartheta(v)=0$, so that $u$ and $v$ are 
$\mathcal{R}$-equivalent elements lying in $G^{\prime}$. But $u$ and $v$ can not be $G$-conjugate since $N u$ and $N v$, having different orders, are certainly not $G / N$-conjugate, and this is against (b) of Lemma 1.4.

Suppose now $G^{\prime} / N \nsucceq A_{5}$. We claim that there exist two elements $N u, N v$ in $G^{\prime} / N$, having distinct orders, and $\chi \in \operatorname{Irr}\left(G^{\prime} / N\right)$, such that $\chi$ and all its $G / N$ conjugates vanish on both $N u$ and $N v$. We shall consider all the possible cases, referring to [4] for the notation of the characters.

Assume that $G^{\prime} / N$ is isomorphic to one of the following groups: $A_{n}$ for $n \geq 8$, $\mathrm{Sp}(6,2), \mathrm{SO}^{+}(8,2)$. Then, as mentioned above, $G^{\prime} / N$ has an irreducible character $\chi$ of 5 -defect zero, and there exist 5 -singular elements $N u, N v$ in $G^{\prime} / N$ having distinct orders: in the case $G^{\prime} / N \simeq A_{n}$ for $n \geq 8$, we can choose $N u=(12345)$ and $N v=(12345)(678)$, whereas the other cases can be easily treated by means of [4]. Now $\chi$ and all its $G / N$-conjugates (which are of 5 -defect zero as well) vanish on $N u$ and $N v$.

A similar argument works if $G^{\prime} / N \simeq \operatorname{PSp}(4,3)$ or $G^{\prime} / N \simeq \operatorname{PSU}(4,3)$, replacing the prime 5 with 3 . Therefore, it remains to treat the case when $G^{\prime} / N$ is isomorphic to one of the groups $A_{6}, A_{7}, \operatorname{PSL}(3,4)$.

Assume $G^{\prime} / N \simeq A_{6}$, and take $\chi=\chi_{7}$, the unique irreducible character of degree 10. This $\chi$ vanishes on elements of order 4 and 5 .

Assume $G^{\prime} / N \simeq A_{7}$, and take $\chi=\chi_{2}$, the unique irreducible character of degree 6 . This $\chi$ vanishes on elements of order 3 and 4 .

Finally, assume $G^{\prime} / N \simeq \operatorname{PSL}(3,4)$, and take $\chi=\chi_{2}$, the unique irreducible character of degree 20. This $\chi$ vanishes on elements of orders 4 and 5 .

The claim is then proved. So, still assuming $G^{\prime} / N \not A_{5}$, let $N u, N v$ and $\chi$ be as required. As above, let $\vartheta$ be an irreducible character of $G / N$ whose restriction to $G^{\prime} / N$ has $\chi$ as a constituent. Then $\vartheta \downarrow_{G^{\prime} / N}$ is a multiple of a sum of $G / N$ conjugates of $\chi$, and so $\vartheta(N u)=\vartheta(N v)=0$. Now, viewing $\vartheta$ as a character of $G$ by inflation, we get $\vartheta(u)=\vartheta(v)=0$. This is a contradiction, as $u$ and $v$ are $\mathcal{R}$-equivalent elements of $G^{\prime}$, but they are not $G$-conjugate because $N u$ and $N v$ are not $G / N$-conjugate.

To complete the proof, we need to exclude the possibility that $G^{\prime} / N$ is isomorphic to $A_{5}$. If it is so, we have $G / N \simeq S_{5}$ (recall that $G / N$ acts faithfully by conjugation on $G^{\prime} / N$, and $G / N$ can not coincide with $G^{\prime} / N$ because $G / N$ must be a rational group, whereas $A_{5}$ is not). Let $u \in G$ be such that $N u=(12)(34)$, and let $v \in G$ be such that $N v=(1234)$. There exists $\chi$ in $\operatorname{Irr}(G)$ of degree 4 , whose kernel contains $N$, which vanishes on both $u$ and $v$. Thus $u$ and $v$ are $\mathcal{R}$-equivalent, and this again violates Lemma 1.4(b).

Proof of the Main Theorem. Let $N$ be a normal subgroup of $G$ properly contained in $G^{\prime}$ (we allow $N=1$ only if $G^{\prime}$ is minimal normal in $G$ ), and let $Z$ denote the subgroup of $G$, containing $N$, such that $Z / N=Z(G / N)$. Our proof is organized as follows. In Case (a) we assume that $Z / N$ is not trivial, whereas in Case (b) we treat the other possibility. As we shall see, in both situations $G^{\prime}$ 
turns out to be minimal normal in $G$ (so $N$ is in fact trivial), and Case (a) yields conclusion (a) of the statement, whereas Case (b) yields conclusion (b).

Case (a) $(Z / N \neq 1)$. Let us assume that $G / Z$ is nonabelian. Then, by Lemma 1.5, $Z$ is minimal normal in $G$, which is impossible if $N \neq 1$. Hence $N$ is trivial, $G^{\prime}$ is minimal normal in $G$, and $G^{\prime} \cap Z=1$. Let $t$ be in $G^{\prime} \backslash\{1\}$ and $z$ in $Z \backslash\{1\}$; if $\psi \in \operatorname{Irr}(G) \backslash \operatorname{Lin}(G)$ is such that $Z \leq \operatorname{ker} \psi$, we have $\psi(z t)=\psi(t)$, whence $z t$ and $t$ are $\mathcal{R}$-equivalent. But $z t$ and $t$ can not be $G$-conjugate, and this contradicts Lemma $1.4(\mathrm{~b})$. Therefore $G / Z$ is abelian, so that $G^{\prime}$ is contained in $Z$ and $G / N$ is nilpotent of class 2. By Lemma 1.5, we also know that $G^{\prime} / N$ is a minimal normal subgroup of $G / N$, and since it is central, its order must be a prime $q$. Now, every element of $G^{\prime}$ is rational in $G$, hence every element of $G^{\prime} / N$ is rational in $G / N$. In other words, if $x$ is in $G^{\prime} \backslash N$ and $s$ is an integer coprime to $q$, then $N x$ and $(N x)^{s}$ are conjugate in $G / N$. We conclude that $G^{\prime} / N$ has order 2 .

Next, as $G / N$ is nilpotent, we write it as $(T / N) \times(R / N)$ where $T / N$ is the (nontrivial) Sylow 2-subgoup of $G / N$, and $R / N$ is the Hall $2^{\prime}$-subgroup of $G / N$. If $G / R$ is abelian, then $G^{\prime} \leq R$ holds, so that the 2-group $G^{\prime} / N$ is a subgroup of $R / N$, a contradiction. Therefore $G / R$ is nonabelian, so that either $R$ is a minimal normal subgroup of $G$, or it is trivial (this can clearly occur only when $N=1$ ). If $N \neq 1$, then $R$ must coincide with $N$. If $N=1$, we get $R \cap G^{\prime}=1$, hence $R$ is central; if it is nontrivial, then its nontrivial elements form a conjugacy class, whence $R$ has order 2 (a clear contradiction). In any case, $G / N$ is a 2 -group.

Our next claim is that $G / N$ is an extraspecial 2-group. Let $\chi$ be a nonlinear irreducible character of $G$ whose kernel $K$ contains $N$. As the quotient $G / K$ is clearly nonabelian, $K$ is trivial or a minimal normal subgroup of $G$. If $K \neq 1$ and $N=1$, then we take $t$ in $G^{\prime} \backslash\{1\}$ and $z$ in $K \backslash\{1\}$, and we get $\chi(z t)=\chi(t)$. Therefore $z t$ and $t$ are $\mathcal{R}$-equivalent, but they can not be $G$-conjugate, against Lemma 1.4(b). We conclude that in any case $K$ coincides with $N$, so that $\chi$ can be regarded as a faithful irreducible character of $G / N$. In particular, $Z / N$ is cyclic. We know that $G^{\prime} / N \leq Z / N$, and we need to show that equality holds. We get $\chi \downarrow_{Z / N}=\chi(1) \mu$, where $\mu$ is a faithful irreducible character of $Z / N$. Let $N a, N b$ be distinct elements of $Z / N$. If $\mu(N a)=\mu(N b)$, then we get $\mu\left(N a b^{-1}\right)=1$, and the faithfulness of $\mu$ yields a contradiction. The conclusion is that $\mu$ takes $|Z / N|$ different values. Since $\chi$ is a rational character, $\mu$ is rational as well, and so the values of $\mu$ lie in $\{-1,1\}$. But this forces $Z / N$ to have order 2 , and the claim is proved.

Now, if $N$ is trivial, we are clearly done. Our assumption will be henceforth $N \neq 1$, and from this we shall derive a contradiction.

Since $N \backslash\{1\}$ is a conjugacy class (and $N$ is abelian), we get that either $|N|=2$, or $|N|-1=\left|G: C_{G}(n)\right|$ is a nontrivial power of 2 , where $n \in N \backslash\{1\}$.

If $|N|=2$, then $N$ is central in $G$. We claim that in fact $Z(G)=N$ holds. We have $Z(G) / N \leq Z / N=G^{\prime} / N$; if $Z(G)$ strictly contains $N$, then we get $Z(G)=G^{\prime}$ (recall that $\left|G^{\prime} / N\right|=2$ ). Now, take $z$ in $Z(G) \backslash N, n$ in $N \backslash\{1\}$, and $\chi$ in $\operatorname{Irr}(G) \backslash \operatorname{Lin}(G)$ such that $\operatorname{ker} \chi \geq N$. We have $\chi(n z)=\chi(z)$ and, since 
$n z$ and $z$ lie in $G^{\prime}$, these two elements must be conjugate in $G$. But $n z$ and $z$ are in $Z(G)$, so they are equal, a contradiction. We are now in a position to apply Theorem $\mathrm{C}$ in $[\mathbf{1 1}]$, obtaining that $G / G^{\prime}$ has order 4 . Now, by a theorem of Taussky ([10, III, 11.9(a)]), $G$ is isomorphic to a quaternion, dihedral or semidihedral group of order 16. But in each of these groups there exist two elements of order 8 on which a nonlinear irreducible character vanishes, whereas another one takes different values (see $[\mathbf{1 3}, 26.8(\mathrm{~A})]$ ), and this contradicts our assumption that all nonlinear irreducible characters of $G$ are $\mathcal{R}$-separators.

Consider now the case when $|N|-1=\left|G: C_{G}(n)\right|$ is a power of 2 different from 1 , so that the prime divisor $p$ of $|N|$ is not 2 . If $|N|=p^{\beta}$, we get $p^{\beta}-1=2^{\alpha}$. If $\beta>1$, then

$$
2^{\alpha}=(p-1)\left(\sum_{i=0}^{\beta-1} p^{i}\right),
$$

whence 2 is a divisor of $\sum_{i=0}^{\beta-1} p^{i}$. The conclusion is that $\beta$ must be an even number, say $2 \gamma$. Now we get $2^{\alpha}=\left(p^{\gamma}-1\right)\left(p^{\gamma}+1\right)$, and therefore one of the two factors is a power of 2 congruent to 2 modulo 4 . Clearly, we now have $p^{\gamma}-1=2$, so that $p=3$ and $|N|=9$. Our argument shows that if $|N| \neq 9$ then $N$ is cyclic of prime order $p$, where $p$ is a Fermat prime.

Next, we have $G \simeq N \rtimes S$, where $S$ is a Sylow 2-subgroup of $G$, and we shall distinguish two situations: $G / C_{G}(N)$ is abelian, or it is not.

In the former case, $S / C_{S}(N)$ is abelian, so that $S^{\prime}$ is contained in $C_{S}(N)$. Moreover, $S^{\prime}$ is contained in $Z(S)$, and the conclusion is that $S^{\prime}$ lies in $Z(G)$. Now, let $z$ be the nonidentity element of $S^{\prime}$, and let $n$ be a nonidentity element of $N$. If $\chi$ is a nonlinear irreducible character of $G$ such that $\operatorname{ker} \chi \geq N$, then $\chi$ takes the same value on $z$ and $n z$. On the other hand, these two elements of $G^{\prime}$ are certainly not $G$-conjugate, against Lemma 1.4(b).

Assume now that $G / C_{G}(N)$ is not abelian. In this setting, $C_{G}(N)$ is minimal normal in $G$, hence it is forced to be $N$, and $S$ acts faithfully on $N$. If $|N|$ is not 9 , then $N$ is cyclic of prime order, so its group of automorphisms is also cyclic. But now $S$ must be cyclic, and this is a contradiction. Finally, let us consider the case $|N|=9$. We get $S \leq \mathrm{GL}(2,3)$, so that $S$ is an extraspecial 2-group which is isomorphic to a subgroup of the semidihedral group of order 16 , and hence $|S|=8$. Moreover, if $n$ is a nonidentity element of $N$, then the conjugacy class of $n$ in $G$ has size 8 , and hence $\left|S: C_{S}(n)\right|=8$. Since $|S|=8$, every nontrivial element of $S$ acts fixed-point freely on $N$, so $S$ is a quaternion group (otherwise it would be cyclic, but we are assuming $S$ nonabelian). Then there exist two elements of $G$, having order 2 and 4 , such that the irreducible character of degree 8 of $G$ vanishes on both, whereas the irreducible character of degree 2 of $G$ takes different values on them. This violates our hypotheses and the analysis of Case (a) is complete.

Case (b) $(Z / N=1)$. Theorem 1.6 ensures the solvability of $G$, and, by Lemma 1.5, $G^{\prime} / N$ is a minimal normal subgroup of $G / N$. Hence we can apply Theorem 1 of $[\mathbf{2}]$, obtaining that $G / N$ is a Frobenius group with kernel $G^{\prime} / N$ 
and cyclic complement. In order to achieve the desired conclusion, we show that $N$ is trivial.

Observe that every nonlinear irreducible character of $G$ whose kernel contains $N$ vanishes off $G^{\prime}$, whence $G \backslash G^{\prime}$ is (contained in) an $\mathcal{R}$-equivalence class. If $G \backslash G^{\prime}$ is a conjugacy class, then by Lemma $1.4(\mathrm{~b})$ the $\mathcal{R}$-equivalence classes in $G \backslash\{1\}$ coincide with the conjugacy classes, and we have that two nontrivial elements of $G$ are conjugate if and only if every nonlinear irreducible character of $G$ takes the same value on them. Theorem 2 of [1], together with Lemma 1.4(c), yields that $G$ is isomorphic to $S_{3}$ in this case, and we are done. Otherwise, there are two elements in $G \backslash G^{\prime}$ which are not conjugate, and by Lemma 1.4(b) it follows that every nonlinear irreducible character of $G$ vanishes off $G^{\prime}$.

For a proof by contradiction, assume $N \neq 1$. Set $|N|=p^{n}$ and $\left|G^{\prime} / N\right|=q^{r}$, where $p$ and $q$ are suitable prime numbers (recall that, by $1.5, G^{\prime} / N$ is a chief factor of $G$ ), and let $t$ be an element of $G^{\prime} \backslash N$. Since $G^{\prime} / N$ is abelian, we have $G^{\prime} / N=C_{G^{\prime} / N}(N t)$, whence $\left|C_{G^{\prime}}(t)\right| \geq\left|G^{\prime} / N\right|$. We claim that $\left|C_{G^{\prime}}(t)\right|=\left|G^{\prime} / N\right|$ holds.

First, we show that for every $x$ in $G^{\prime} \backslash N$ the $G$-conjugacy class of $x$ is a union of cosets of $N$ in $G^{\prime}$. In fact, let $n$ be in $N$, and let $\chi$ be a nonlinear irreducible character of $G$ whose kernel contains $N$. Then we get $\chi(n x)=\chi(x)$, so that $n x$ and $x$ are $\mathcal{R}$-equivalent, whence $G$-conjugate by Lemma 1.4(b).

Next, let us assume $p \neq q$. Then $N$ has a complement $Q$ in $G^{\prime}$, and we claim that every nontrivial element of $Q$ acts fixed-point freely by conjugation on $N$. In fact, let $x$ be in $Q \backslash\{1\}$, and let $n$ be in $C_{N}(x)$. By the discussion in the above paragraph, $n x$ and $x$ must be $G$-conjugate, and this forces $n$ to be trivial (otherwise $n x$ and $x$ would have different orders). The conclusion is that $G^{\prime}$ is a Frobenius group with kernel $N$ and complement $Q$ isomorphic to $G^{\prime} / N$. It follows at once that the desired conclusion $\left|C_{G^{\prime}}(t)\right|=\left|G^{\prime} / N\right|$ holds in this case.

On the other hand, let us assume $p=q$. As $\left|G^{\prime} / N\right|$ is coprime to $\left|G / N: G^{\prime} / N\right|$, we have that $p$ is not a divisor of $\left|G / N: G^{\prime} / N\right|=\left|G / G^{\prime}\right|$. Thus we get $G=G^{\prime} \rtimes H$, where $H$ is a Hall $p^{\prime}$-subgroup of $G$. Denote by $m$ the positive integer such that the size of the conjugacy class of $t$ is $m|N|$; also, for a given integer $i$, let $i_{p}$ denote the greatest power of $p$ which divides $i$. We have $\left|C_{G}(t)\right|=\left(\left|G^{\prime}\right||H|\right) /(m|N|)$, whence

$$
\left|C_{G^{\prime}}(t)\right|=\left|C_{G}(t)\right|_{p}=\left(\left|G^{\prime}\right|_{p}|H|_{p}\right) /\left(m_{p}|N|_{p}\right)=\left|G^{\prime}\right| /\left(m_{p}|N|\right) \leq\left|G^{\prime} / N\right|,
$$

and our claim is proved in this case as well.

What we got so far, together with the Second Orthogonality Relation, and with the fact that $N$ is $G^{\prime \prime}$, yields that every nonlinear irreducible character of $G^{\prime}$ vanishes off $N$. Now, consider an irreducible character $\psi$ of $G$ whose restriction to $G^{\prime}$ has a nonlinear irreducible constituent. We get $\psi(t)=0$ for all $t$ in $G^{\prime} \backslash N$ but, as observed in the second paragraph of Case (b), $\psi$ vanishes also on every element in $G \backslash G^{\prime}$. This is against the second statement of Lemma 1.4(b), and also the analysis of Case (b) is complete.

This completes the proof of the Main Theorem. 


\section{ORDER SEPARATORS AND CLASS-SIZE SEPARATORS}

As an application of the discussion developed in Section 1, we shall consider the following equivalence relations $\mathcal{O}$ and $\mathcal{S}$ on the set of nontrivial elements of a group $G$.

We set $x \mathcal{O} y$ if and only if $x$ and $y$ have the same order, whereas $x \mathcal{S} y$ if and only if $x$ and $y$ lie in conjugacy classes of the same size (it is clear that $\mathcal{O}$ and $\mathcal{S}$ are rational equivalence relations in the sense of Definition 1.3).

Then, as in the Introduction, define an order function to be an $\mathcal{O}$-function, and a class-size function to be an $\mathcal{S}$-function. Of course we define the concepts of order separator and class-size separator accordingly.

We are now in a position to prove Theorems A and B.

Proof of Theorem A. By the Main Theorem, we know that $G$ is either an extraspecial 2-group, or a Frobenius group with kernel $G^{\prime}$ (which is minimal normal in $G$ ) and cyclic complement $H$, depending on whether the centre of $G$ is not trivial or trivial respectively.

In the former case, by Lemma 1.4(b) we have that the nontrivial element of $G^{\prime}$ is the unique involution in $G$. Since $G$ is an extraspecial 2 -group, it follows that $G$ is isomorphic to the quaternion group of order 8 , and (a) holds.

In the latter case, as all the elements in $G^{\prime} \backslash\{1\}$ have the same order $p$, they must be conjugate in $G$. This implies that the order of $H$ is $\left|G^{\prime}\right|-1$. Moreover, the unique nonlinear irreducible character of $G$ vanishes off $G^{\prime}$, whence the nontrivial elements of $H$ are forced to have the same order. The conclusion is that $|H|=q$, where $q$ is a prime. Also, if $\left|G^{\prime}\right|=p^{\alpha}$, we get $q=p^{\alpha}-1$. Now, either $q=2$, $p=3$ and $\alpha=1$, so $G \simeq S_{3}$, or $p=2$ and $G$ is as in (c) (see [10, II, 3.10]). As for the last part of the statement, this is a straightforward check.

Proof of Theorem B. If $Z(G) \neq 1$, then $G$ is as in (a) by the Main Theorem. So we only need to consider the case when the centre of $G$ is trivial. Again by the Main Theorem, $G$ is a Frobenius group with kernel $G^{\prime}$ and cyclic complement $H$. Also, $G^{\prime}$ is minimal normal in $G$, hence an elementary abelian $p$-group for a suitable prime $p$. The elements of $G^{\prime} \backslash\{1\}$ are partitioned into conjugacy classes of the same size $|H|$, so they are forced to lie in a single conjugacy class. Then we get $|H|=\left|G^{\prime}\right|-1$, and conclusion (b) holds (see [10, II, 3.10]). The last part of the statement is a straightforward check.

\section{REFERENCES}

[1] M. Bianchi, A. Gillio, D. Chillag, Finite groups with many values in a column or a row of the character table, Publ. Math. Debrecen 69 (2006), 281-290.

[2] M. Bianchi, A. Gillio, M. Herzog, G. Qian, W. Shi, Characterization of non-nilpotent groups with two irreducible character degrees, J. Algebra 284 (2005), 326-332.

[3] D. Chillag, A. Mann, Nearly odd-order and nearly real finite groups, Comm. Algebra 26 (1998), 2041-2064. 
[4] J.H. Conway, R.T. Curtis, S.P. Norton, R.A. Parker, R.A. Wilson, Atlas of finite groups, Clarendon Press, Oxford, 1985.

[5] W. Feit, G.M. Seitz, On finite rational groups and related topics, Illinois J. Math. 33 (1989), 103-131.

[6] P. Fitzpatrick, Order conjugacy in finite groups, Proc. Roy. Irish Acad. Sect. A 85 (1985), $53-58$.

[7] A. Granville, K. Ono, Defect zero p-blocks for finite simple groups, Trans. Amer. Math. Soc. 348 (1996), 331-347.

[8] M. Herzog, J. Shönheim, On groups of odd order with exactly two non-central conjugacy classes of each size, Arch. Math. (Basel) 86 (2006), 7-10.

[9] B. Huppert, Character Theory of Finite Groups, De Gruyter, Berlin, 1988.

[10] B. Huppert, Endliche Gruppen I, Springer, Berlin, 1983.

[11] I.M. Isaacs, Derived subgroups and centers of capable groups, Proc. Amer. Math. Soc. 129 (2001), 2853-2859.

[12] I.M. Isaacs, Character Theory of Finite Groups, Academic Press, New York, 1976.

[13] G. James, M. Liebeck, Representations and Characters of Groups, Cambridge University Press, Cambridge, 1993.

[14] R. Knörr, W. Lempken, B. Thielke, The $S_{3}$-conjecture for solvable groups, Israel J. Math. 91 (1995), 61-76.

[15] J.P. Zhang, Finite groups with many conjugate elements, J. Algebra 170 (1994), 608-624.

Mariagrazia Bianchi, Dipartimento di Matematica "Federigo Enriques"

Università degli Studi di Milano, Via C. Saldini 50, 20133 Milano, Italy

E-mail address: bianchi@mat.unimi.it

David Chillag, Department of Mathematics

Technion, Israel Institute of Technology, Haifa 32000, Israel

E-mail address: chillag@techunix.technion.ac.il

Emanuele Pacifici, Dipartimento di Matematica "Federigo Enriques" Università degli Studi di Milano, Via C. Saldini 50, 20133 Milano, Italy

E-mail address: pacifici@mat.unimi.it 